\title{
Superbugs: The Nightmare Bacteria
}

\author{
Barkha Sharma $^{1 *}$, Mukesh Kumar Srivastava ${ }^{2}$, Parul $^{3}$, Meena Gosvami ${ }^{4}$ and Udit Jain ${ }^{3}$ \\ ${ }^{1}$ Department of Veterinary Epidemiology and Preventive Medicine, U.P. Pandit Deen Dayal Upadhayaya Pashu Chikitsa Vigyan \\ Vishwavidyalaya Evam Go-Anusandhan Sansthan (DUVASU), Mathura, INDIA \\ ${ }^{2}$ Department of Veterinary Medicine, U.P. Pandit Deen Dayal Upadhayaya Pashu Chikitsa Vigyan Vishwavidyalaya Evam Go- \\ Anusandhan Sansthan (DUVASU), Mathura, INDIA \\ ${ }^{3}$ Department of Veterinary Public Health, U.P. Pandit Deen Dayal Upadhayaya Pashu Chikitsa Vigyan Vishwavidyalaya Evam \\ Go-Anusandhan Sansthan (DUVASU), Mathura, INDIA \\ ${ }^{4}$ Department of Livestock Product Technology, U.P. Pandit Deen Dayal Upadhayaya Pashu Chikitsa Vigyan Vishwavidyalaya \\ Evam Go-Anusandhan Sansthan (DUVASU), Mathura, INDIA
}

"Corresponding author: B Sharma; E-mail: barkha16vet@gmail.com

Received: 15 Dec., 2020

Revised: 20 Aug., 2021

Accepted: 28 Aug., 2021

\begin{abstract}
One of the major achievements in medicine was in 1928 when the first antibiotic penicillin was discovered by Alexander Flemming. They were touted as 'Miracle drug' and brought great promise of a future without infectious disease. And now we face the threat of superbugs or nightmare bacteria threatening to render our future bleak. Each year, Anti Micorbial Resistance (AMR) kills more than 700,000 people globally. A 'superbug' is a bacterium capable of causing uncontrollable infections, a microbe which can't be eradicated, and a germ which can kill when normally it shouldn't. These are no less than "nightmare bacteria" that have a potential to threaten people in every nook and corner of the world catastrophically. Widespread use of antibiotics without good stewardship has eventually led to emergence of multiple drug resistance against almost all of our life saving antimicrobial drugs including the last resort wonder drug colistin. Thus, if we still do not face this problem head on and make some strict decisions, we may be looking at a post antibiotic era marked with minor wounds becoming life threatening and people dying from simple infections.

\section{Highlights}

(0 Superbug is a bacterium that has become resistant to the conventional treatment of antibiotics and is capable of causing uncontrollable infections in humans as well as in animals.

- Main superbugs are MRSA, VRSA, CRPA, CRAB, ESBLs and resistant Mycobacteria causing MDR and XDR TB.

0 India has become notorious as the antibiotic resistance capital of the world.
\end{abstract}

Keywords: Antimicrobial resistance AMR, superbug, Methicillin Resistant Staphylococcus aureus MRSA, Carbapenemresistant Enterobacteriaceae (CRE), Carbapenem Reisistant Pseudomonas aeruginosa (CRPA), Carbapenem-resistant Acinetobacter baumannii (CRAB)

Superbug is a colloquial term used to describe a bacterium that has become resistant to the conventional treatment of antibiotics. A 'superbug' is a bacterium capable of causing uncontrollable infections, a microbe which can't be eradicated, and a germ which can kill when normally it shouldn't. As more and more bacteria become resistant to our existing arsenal of antibiotics, the infections become harder and harder to treat, in some cases rendering a simple bacterial infection deadly. The first ever most comprehensive report on AMR threat with data compiled

How to cite this article: Sharma, B., Srivastava, M.K., Parul, Gosvami, M. and Jain, U. (2021). Superbugs: The Nightmare Bacteria. J. Anim. Res. 11(05): 765-773.

Source of Support: None; Conflict of Interest: None 
from 114 countries, released by WHO puts this threat to be one of the most glaring global health threats of the $21^{\text {st }}$ century (WHO, 2014). These superbugs are responsible of causing deaths of nearly 1 million people due to non treatable drug resistant infections every year worldwide (Ventola, 2015). These figures are expected to rise upto 10 million by mid-century, putting the threat due to AMR in same category as the HIV/AIDS crisis, calling for a coordinated response from the international community (Healio, 2016).

To support and strengthen the global antimicrobial surveillance and to achieve the goals adopted by World Health Assembly in May, 2015, WHO launched the Global Antimicrobial Surveillance System (GLASS) in October same year. The main objective of GLASS is to facilitate a standardized approach to combat the widespread occurrence of antibiotic resistance across 22 countries and share the data at global level (GHL, 2018). Former UK PM David Cameron called the fight against drugresistant infections the "perfect example of something that demands international action." These superbugs are no less than "nightmare bacteria" that have a potential to threaten people in every nook and corner of the world catastrophically.

In 1928, one of the major milestones in medicine was achieved when the first antibiotic penicillin was discovered by Alexander Flemming. They were touted as 'Miracle drug' and brought great promise of a future without infectious disease. The life expectancy increased from 50 years in 1945 to 80 years in the $21^{\text {st }}$ century (Schmidt et al., 2007). Infections such as syphilis, diarrheal illnesses, and lower respiratory tract infections were no longer considered to be fatal and were easily cured. Flemming's contribution to humanity changed the course of modern medicine but during his acceptance speech at the Nobel Prize Award presentation, he warned that widespread use of antibiotics without good stewardship would eventually cause resistance and in some instances, multiple drug resistance. And this is what is eventually happening in real time. Gram negative bacteria are most often resistant to antimicrobials as a result of their production of enzymes like beta lacatamases along with natural up-regulated impermeability and efflux (Livermoore and Paterson, 2006). In gram-positive cocci like pneumococci and MRSA, most common reason is target modification or change in the normal Penicillin Binding Proteins (PBPs) or acquisition of additional $\beta$-lactam-insensitive PBPs.

\section{Antimicrobial Resistance (AMR): cause and the effect}

Globally, antibiotics are the most abused medications. Data says almost two-third of the antibiotics used all over the world is inappropriate or unnecessary (McKenna, 2016). The yelp effect (overprescribing for patient satisfaction), incorrect regimen (over/under medication), wrong duration, over the- counter availability of scheduled drugs, prescribing antibiotics for viral infections and inability to stick to the prescribed dosage are deemed to be major causes of antibiotic abuse (Abdulah, 2012). In developing countries, this might be attributed to lack of knowledge, self medication, and dependence on unqualified quacks. Preference to prescribe newer broad-spectrum antibiotics without diagnosing the underlying illness is exacerbating the problem. Infact, this overuse or misuse of antibiotics is responsible for causing more morbidity than cancer. Each year, AMR kills more than 700,000 people globally. The inappropriate drug usage in human medicine, animal husbandry, and agriculture also brings about phenotypic changes in pathogens due to chromosomal mutations (Walsh and Wright, 2005). The speed with which most of the pathogens are becoming resistant to the available antimicrobials is alarming and is undoubtedly having major negative impact on veterinary and human medicine (Heiko and Reinhardt, 2005)

Antibiotics are rightly called the world's first blockbuster drugs as they revolutionized the way infections were treated, saving lives and ensuring profits in the decades after World War II. However, with the rise of Multidrug Resistant Organisms (MROs), or alleged superbugs, bacteria have not only kept up with, but often outpaced whatever scientific progress we have made. Whenever an antibiotic is given, it is intended to kill all the target organisms but some escape treatment, develop resistance and propagate their own kind. These superbugs are not only armed with better survival strategies, they keep expanding their tribe by transferring resistance genes through "horizontal gene transfer" (direct transfer of genetic material from one organism to another without any reproduction) to non resistant bacteria, making them resistant in turn. Antibiotic resistance is a continuous and inevitable process. Antimicrobial resistant organisms are present all around us, in food we eat, water we drink and the environment we live. We are now actually realizing the scale of this gargantuan problem. 
The story doesn't end here. AMR is spreading through various indirect ways too. Worldwide, much higher volumes of antibiotics are used in food animals than in human medicine. The routine prophylactic massmedication with antibiotics is common practice especially in pig and poultry sectors in intensive farming systems. Majority of all the antibiotics produced are given to livestock in the developed countries like UK, EU, and USA. There is a high probability of the spread of resistant strains of bacteria to humans consuming the animal products and thus healthy individuals can become carrier hosts for multiple antibiotic-resistant bacteria (Reinthaler et al., 2003). There are several reports of surface water bodies being contaminated with resistant bacteria originating from various human and livestock sources (Harakeh et al., 2006).

With the crisis in full toddle, the Centers for Disease Control and Prevention (CDC) developed a system to classify the seriousness of antibiotic resistance in 2013 with major focus on the urgent and serious threats, which require close monitoring and prevention activities to protect against an apocalyptic event.

Table 1: WHO Priority List for AMR bacteria

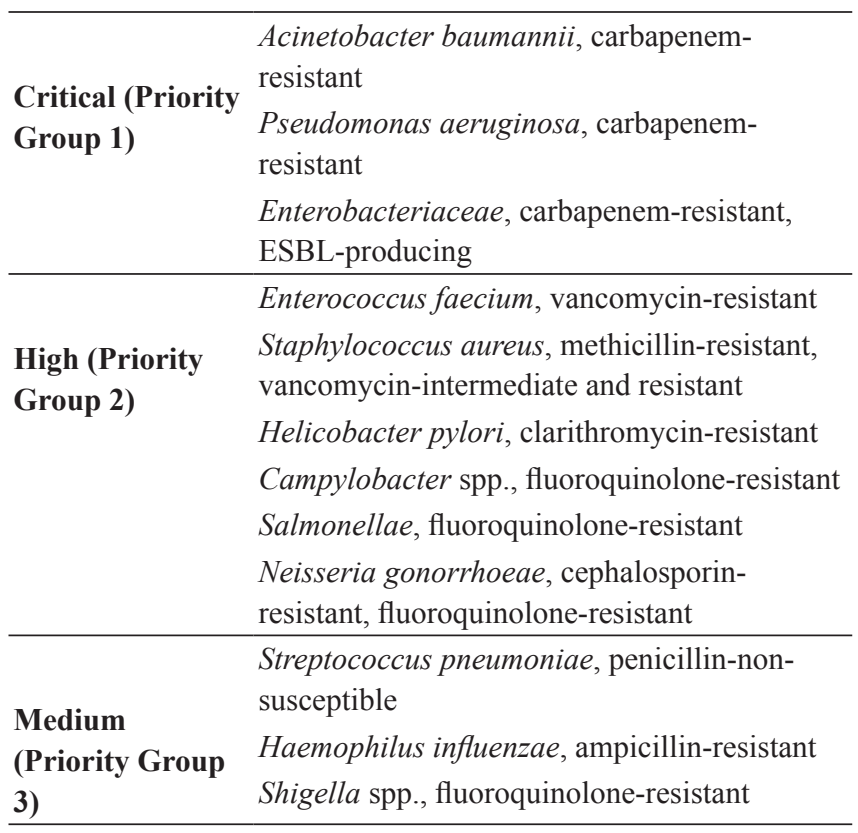

Source: WHO website (https://www.who.int/medicines/publications/ global-priority-list-antibiotic-resistant-bacteria/en/).
Table 2: CDC Priority List for AMR bacteria

\begin{tabular}{ll}
\hline Urgent & Clostridium difficile \\
Threats & Carbapenem-resistant Enterobacteriaceae (CRE) \\
& Drug-resistant Neisseria gonorrhoeae \\
\hline Serious & Multidrug-resistant Acinetobacter \\
Threats & Drug-resistant Campylobacter \\
& Fluconazole-resistant Candida \\
& Extended spectrum $\beta$-lactamase producing \\
& Enterobacteriaceae (ESBLs) \\
& Vancomycin-resistant Enterococcus (VRE) \\
& Multidrug-resistant Pseudomonas aeruginosa \\
& Drug-resistant Non-typhoidal Salmonella \\
& Drug-resistant Salmonella Typhi \\
& Drug-resistant Shigella \\
& Methicillin-resistant Staphylococcus aureus \\
& (MRSA) \\
& Drug-resistant Streptococcus pneumoniae \\
& Drug-resistant tuberculosis \\
\hline Concerning & Vancomycin-resistant Staphylococcus aureus \\
& (VRSA) \\
& Erythromycin-resistant Group A Streptococcus \\
Threats & Clindamycin-resistant Group B Streptococcus \\
\hline
\end{tabular}

Source: https://www.cdc.gov/drugresistance/threat-report-2013/ pdf/ar-threats-2013-508.pdf

According to USFDA, almost $70 \%$ of bacteria have become resistant to antibiotics globally, including our last resort drugs. With our arsenal to fight the majority of bacterial infections almost depleted as AMR spreads, we are heading towards a "post antibiotic era" where common infections and minor injuries could once again become life threatening. The ones most vulnerable to a "superbug" infection are immune compromised patients receiving chemotherapy, individuals who have undergone complex surgeries or patients afflicted with chronic diseases such as diabetes, asthma, and rheumatoid arthritis. The greatest numbers of deaths from these "superinfections" occur in the hospitals and nursing homes.

The AMR bacteria have been defined as multidrugresistant (MDR), extensively drug-resistant (XDR), and pandrug-resistant (PDR) (Magiorakos et al., 2012). MDR is acquired nonsusceptibility to at least one agent in three or more antimicrobial categories. Extensively drug resistant (XDR) is nonsusceptibility to at least one agent in all but two or fewer antimicrobial categories (i.e., 
bacterial isolates remain susceptible to only one or two antimicrobial categories) and Pandrug resistant (PDR) is nonsusceptibility to all agents in all antimicrobial categories.

According to the "Antimicrobial resistance: global report on surveillance" released by WHO in 2014, although many infectious agents show AMR, the major players are the seven different bacteria responsible for common, serious diseases such as sepsis, diarrhoea, pneumonia, urinary tract infections and gonorrhea like methicillin- resistant Staphylococcus aureus (MRSA), carbapenem resistant Klebsiella pneumonia etc.

In this review, a brief summary of the most common superbugs has been compiled.

\section{Carbapenem-resistant Acinetobacter baumannii (CRAB)}

Highly-drug resistant Gram-negative bacteria that have become resistant to virtually all antibiotics capable of treating Gram-negative bacteria, including the fluoroquinolones, cephalosporins, and carbapenems, the resistance rates exceeding more than $90 \%$ in some parts of the world (WHO, 2017). CRAB may be extensively drugresistant (XDR) or pandrug-resistant (PDR) (Tacconeli et al., 2018) and is constantly gaining the notoriety of being one of the most untreatable pathogen of all. The acquired resistance genes (inherent class D $\beta$-lactamase gene (blaOXA-51-like, blaOXA-23 and blaOXA-58) get accumulated in large resistance islands called as hot-spot within the bacterial chromosome. CRAB has evolved as a significant hospital pathogen by its ability to resist desiccation, disinfectants, and major antimicrobials (Peleg et al., 2008) and can cause a range of infections in hospitalized patients, including pneumonia, wound, or blood infections with mortality rates for the most common hospital-acquired pneumonia (HAP) and bloodstream infections (BSI) approaching 60\% (Wong et al., 2017). The infections caused by CRAB are not treated by any other drug except colistin which is the last remaining option. This has made it certain to be included in the notorious company of the likes of MRSA or Clostridium difficile (Evans et al., 2013).

\section{Carbapenem Reisistant Pseudomonas aeruginosa (CRPA)}

Pseudomonas aeruginosa can cause skin rashes and ear infection in healthy people but also severe blood infections and pneumonia in immunocompromised people especially in hospital settings. It is one of the hardest bacteria to eradicate. First isolated in 2001 in the USA, Verona Integron-Mediated (VIM) metallo-beta-lactamase is currently the most common type of carbapenemase seen in $P$. aeruginosa worldwide. In 2010 , metallo- $\beta$ lactamases (MBL) carbapenemases were detected in the U.S. The gene encoding these carbapenemases are present on mobile genetic elements that can jump from one strain of bacteria to other and across the species, spreading the resistance far and wide, making them a serious public health threat. CRPA spreads rapidly in health care settings through contact with contaminated surfaces and patient care equipment or contact between patients and health care workers. These bacteria are not spread through the air.

\section{Extended-spectrum beta-lactamases (ESBL) producing Enteriobacteriaceae}

ESBL are transmissible $b$ lactamases that confer resistance to most beta-lactam antibiotics. Resistance to beta lactams antibiotics emerged within 5 years after their introduction in Europe in 1960s. The first ESBL was called TEM after the patient from whom it was isolated (Temoniera) (Paterson and Bonomo, 2005). Now there are more than 400 ESBLs clustered into 9 different structural and evolutionary families. The ESBL genes are located on plasmids easily transferrable between bacteria (Coque et al., 2008). The most common ESBL producing enteriobacteriacae are Klebsiella pneumoniae, E coli, and Proteus mirabilis. Recently, there has been a spate in the incidence of ESBL- related infections throughout the globe (Rupinder et al., 2013). Klebsiella pneumoniae is normally found in $3-5 \%$ of the population but can cause life-threatening nosocomial infections such as pneumonia, bloodstream infections in immunocompromised patients and newborns. E.coli are often implicated in urinary tract infections. Multiple subclones of an extensively antimicrobial-resistant $E$. coli sequence type 131 (ST131) 
viz., H30, H30-R, and H30-Rx have spread explosively throughout the world, of which $\mathrm{H} 30$ is the most prevalent (Banerjee and Johnson, 2014; Tchesnokova et al., 2019). Hitherto carbapenems and fluoroquinolones have been the main stay of treatment against ESBL bacteria but resistance against them is increasingly being reported from all over the world. Now Colistin is the only last resort treatment against carbapenem resistant Enterobacteriaceae (CRE) but resistance to it has also been detected in several countries and regions, leaving us with no effective antibiotic treatment for the infections caused by CRE at present.

\section{Methicillin Resistant Staphylococcus aureus (MRSA)}

Staphlylococcus aureus is found in our skin flora and implicated as a common cause of community-spread and nosocomial infections. It was in 1950s when some strains of Staphylococcus aureus reported resistance to penicillin and within a year of introduction in 1959, resistance developed against Methicillin too. These resistant strains were known as 'methicillin-resistant Staphylococcus aureus', or 'MRSA'. Though MRSA is being reported for almost half a century now, but has only lately become a cause of deep public anxiety, especially among those faced with admission to hospital. MRSA resides in human tissues and eventually becomes resistant when not handled properly (Christoff et al., 2001). Initially, MRSA caused minor skin infections like pimples, spider bites or boils that may later on become purulent, painful, swollen, or warm to the touch, causing fever. A few progress and spread further into the skin and to other areas or organ systems. People with MRSA have 64\% more propensity to death compared to people with a non-resistant form of the infection.

\section{Clostridium difficile}

Clostridium difficile has evolved as a superbug due to antibiotics being prescribed to the patients over long periods of time in extended or repeated regimens. Overdosing of antibiotics disrupts gut flora and helps to form a conducive environment for the $C$. difficile bugs to multiply and take over, resulting a seemingly innoucuous infection to turn lethal. Clostridium difficile infection (CDI) is the most frequent hospital-acquired intestinal infection in Europe and worldwide (O'Donoghue and Kune, 2011). The survival of the bacteria in the presence of antibiotic is aided by a plethora of factors like acquisition of genetic elements, alterations of the antibiotic target sites, variations in the metabolic pathways and biofilm production (Spigaglia et al., 2018). Recent data indicate that even in absence of antibiotic selective pressure, resistances may persist in $C$. difficile population as the development and maintenance of antibiotic resistance in this bacterium is aided by several mechanisms. Almost $11 \%$ of the $C$. difficile genome is made of mobile genetic elements (MGEs) or transposons. Any alterations in the antibiotic targets can be quickly passed within the cells and maintained in the population. Relapses are common due to the germination of spores of $C$. difficile leading to treatment failure. The ribotype 027 of $C$. difficile has been found responsible for increasing number of outbreaks since the early 2000s, in turn increasing the medical cost throughout the world along with higher mortality (Peng et al., 2017).

\section{Drug resistant Tuberculosis}

Tuberculosis is a chronic disease that occurs worldwide and needs no introduction. It is a scoorge for socioeconomically weaker sections of society as well as immunocompromised patients and newborns. It usually affects the lungs but brain, kidneys or spine may also be affected. Drug resistant TB accounts for more than 1.7 million deaths worldwide annually. In the 1940 s, multidrug therapy with a combination of 4 drugs (at least 2 active drugs) begun to be used to prevent relapse and emergence of resistance, resolution of symptoms and microbiological clearance of Mycobacterium but resistance emerged in no time. Multi-drug-resistant TB (MDR-TB) is unresponsive to first and second line of antitubercular drugs especially isoniazid (INH) and rifampin. XDR-TB is defined as MDR-TB with additional resistance to the third line of drugs i.e., any fluoroquinolone and to at least one of three injectable agents (kanamycin, amikacin, or capreomycin). In 2013, there were almost 9 million TB cases all over the world, of which $3.5 \%$ were of MDR-TB amounting to 480,000 cases. Extensively drug-resistant TB (XDR-TB) was reported from around 100 countries and almost 9\% of people with MDR-TB tend to progress to have XDRTB according to Global Tuberculosis Report by the World Health Organization (WHO). 


\section{Drug Resistant Neisseria gonorrhoeae}

Neisseria gonorrhoeae causes a sexually transmitted disease (STD) called Gonorrhea which manifests varied symptoms in both men and women including infertility. In 2016, approximately 87 million new cases occurred worldwide mainly affecting persons in age group 15-49 years with a global rate of 20 per 1000 women and 26 per 1000 men according to WHO. The African region has the maximum cases of gonorrhea till date. Sulphonamides were once the mainstay of treatment against the bacteria but resistance developed rapidly to sulphonamides, penicillins, tetracyclines, macrolides, fluoroquinolones, and early generation cephalosporins. After that, third generation cephalosporins have been the last resort of treatment but treatment failure to those drugs too has been reported in various European countries, Australia, Canada etc. Neisseria gonorrhea has shown extraordinary inherent predisposition to develop resistance to all antimicrobials owing to its capacity to alter its DNA through mutations. Most AMR genes are located in the chromosome of the bacteria except the genes for resistance against penicillin $\left(\right.$ bla $\left._{\mathrm{TEM}}\right)$ (Phillips, 1976) and tetracycline $\left(\right.$ tet $\left._{\mathrm{M}}\right)$ (Morse et al., 1986). N. gonorrhoeae can also control the concentration of antibiotic entering into the bacterial cell (Suay-Garcia and Pérez-Gracia, 2017). This widespread resistance has made the management and control of gonorrhea an almost impossible task. Currently, in most countries, the injectable extended-spectrum cephalosporin (ESC) ceftriaxone is the only treatment protocol for gonorrhea which is still effective.

\section{The Indian Scenario}

Antimicrobial resistance (AMR) has emerged as a major and continued threat to public health. It is stipulated to cause nearly 10 million deaths annually by 2050 . India is one of the most severely affected countries from drugresistant pathogens as it is supposed to be the home to the most dangerous antibiotic-resistant bugs of the century, some of which are even resistant to colistin, the most potent and toxic antibiotic ever known to mankind. Being the second most populous nation on earth, India is one of the largest markets of antibiotics worldwide, and due to the over the counter availability, the sale of antibiotics is increasing unchecked. Antibiotic abuse is rampant in livestock and agriculture field, food industry and human and animal healthcare, lax regulatory and healthcare policies, poor sanitation, long and improper treatment regimens and unawareness among general public has given India the ignominious title of the antibiotic capital of the world.

In 2008, a bacteria containing an enzyme known as NDM1 (New Delhi metallo-beta-lactamase-1) was found in a Swedish patient who visited India thus the protein was named as NDM-1, after India's capital. The presence of NDM-1 gene makes a bacteria very powerful superbug as it enables the bacteria to produce carbapenemase enzyme. NDM-1 and another similar protein called KPC have been touted as emerging issues in the field of infectious diseases by CDC. The plasmid containing the NDM-1 gene can jump between bacteria by horizontal gene transfer (HGT). This gene has become endemic in India, widely found in nosocomial environment or drinking water and the holy rivers such as the Ganges. Factors that led to the emergence of NDM-1 in India are still not clear. The warm and humid climate might encourage its persistence year round. Since then by 2015 , the bacteria expressing NDM1 have surfaced in more than 70 countries worldwide including the United States, Japan, Australia, and the United Kingdom.

India poses a grand challenge for controlling AMR because it is the largest consumer of antimicrobials globally (Kakkar et al., 2017; WHO, 2015), due to ease of availability of scheduled medications for both humans and livestock. Indians becoming more and more resistant to all the antibiotics in general and some of the precious few drugs in the reserve category like colistin which is being used to fatten the chickens in poultry farms. However, slowly but steadily, India is gearing up to fight against AMR. Kerala was the first Indian state to formulate and execute an antimicrobial resistance (AMR) action plan followed by promulgation of first National Action Plan to combat antimicrobial resistance in 2017 by the government of India. The plan focuses on curbing rampant antibiotics misuse in health and agriculture field, encouraging rational use of antibiotics in hospitals and the community and strengthening surveillance. India's agricultural ministry also recognizes the dangers of widespread misuse of antibiotics in animal feed and supplements. Oxytetracycline, Doxycycline, Ciprofloxacin and Neomycin have been recognized as antibiotics most commonly detected in tissues of marketed poultry. 
A lot remains to be done. There's an immediate need to wean off our livestock from unnecessary antibiotics given to enhance their production and longetivity. A shift to organic farming methods might also help in reducing the usage of excess antibiotics in livestock (Zwald et al., 2004). There should be tighter restrictions on OTC sale of antibiotics and stricter implementation of such laws already in place. Stricter rules should be made to check the effluent drainage from hospitals and drug manufacturing plants in water sources which create favorable conditions for the growth of MDR bacteria. Stringent hygiene and sanitation policies should be made for the proper disposal of medical waste. The general public should be made aware of the hazards of buying drugs without a proper prescription from a verified doctor. Medical and veterinary fraternity should be made accountable for the antibiotics they prescribe for treatment. There's is an immediate need to wake from a long slumber and start mobilizing ourselves. The war is just beginning.

\section{CONCLUSION}

The AMR is all around us, in our hospitals, on our farms and in our food, too. While the threat of superbugs is deeply alarming, strong and conscious efforts are being done to combat these infections. From innovative laboratory based research to the surveillance and monitoring, development and implementation of sustainable and effective policies, efforts are needed in every imaginable field to help us protect antibiotics as the lifesaving resource they are. European Union and USA already put a ban on the usage of antibiotics as growth promoters in 2006 and 2017, respectively. They have also been trying to make the public aware of the disadvantages of using excess antibiotics by various public awareness programmes.

Onus also lies on the agriculture sector to share the responsibility, both by using antimicrobials more judiciously and adopting good farm hygiene practices. Environment serves as a major reservoir for antibiotic resistant genes. Soil itself is source of many bacteria that produce antimicrobial compounds. All the antibiotics used as growth promoters, feed additives, fertilizers etc eventually find their way back to human and animal bodies and cause more damage. This vicious cycle continues till one of the elements of the cycle is removed deliberately.
Even WHO and European Food Safety Authority (WHO, 2011) support this theory.

Knowledge is power, so the first step towards an AMR free world is acquiring authentic data regarding the actual extent of spread, cause of spread and potential methods for control and prevention. Global surveillance systems like The Study for Monitoring Antimicrobial Resistance Trends (SMART) might be a step forward in this path. Antimicrobial Stewardship Programs (ASPs) will help in understanding the need for a fundamental shift away from routine reliance on antibiotics in every field. Educating the general public not to misuse antibiotics by preventing their OTC usage as well as making sure that clinicians and veterinarians do not over or under prescribe the drugs is essential for the Success of ASPs. We have an uphill task on our hands. We need a system-wide approach to tackle this behemoth problem. Significant role has to be played by all the stakeholders be it livestock farmers, producers, food retailers, pharmaceutical companies and policymakers at local, regional, national and global level if we have to achieve our goals. Antibiotics are not recreational drugs. Their usage should be restricted to instances where they serve as life savers. Making appropriate laws and strict regulatory scrutiny is urgently needed. In order to address the importance of unchecked rising of AMR and to bring this into the notice of general public, the "Global Action Plan" was endorsed by the World Health Assembly in May 2015, laying down the guideline to combat AMR by putting in place action plans at national and international levels within 5-10 years to ensure that the future generations may not have to live in an uncertain world devoid of the major arsenals to fight the infectious diseases, the life saving drugs, the wonder drugs, the antibiotics.

\section{REFERENCES}

Abdulah, R. 2012. Antibiotic abuse in developing countries. Pharm. Regul. Aff., 1: 1000-1106.

Banerjee, R. and Johnson, J.R. 2014. A new clone sweeps clean: the enigmatic emergence of Escherichia coli sequence type 131. Antimicrob. Agents Chemother, 58(9): 4997-5004.

Christof, V.E., Becker, K., Machka, K., Stammer, H. and Peters, G. 2001. Nasal carriage as a Source of Staphylococcus aureus bacteremia Study Group. N. Engl. J. Med., 344: 11-16. 
Coque, T.M., Baquero, F. and Cantón, R. 2008. Increasing prevalence of ESBL-producing Enterobacteriaceae in Europe. Euro. Surveill., 13: 1.

Evans, B.A., Hamoude, A. and Amyes, S.C. 2013. The rise of carbapenem-resistant Acinetobacter baumanni. Curr. Pharm. Des., 19(2): 223-28.

Global Health Link. 2018. Superbugs: how the misuse of antibiotics has threatened communities around the globe. 55.

Harakeh, S., Yassine, H. and El-fadel, M. 2006. Antimicrobial resistance pattern of Escherichia coli and Salmonella strains in the aquatic Lebanese environments. Environ. Poll., 143: 269.

Healio - Infectious Disease News. 2016. Experts call for response to antibiotic resistance similar to AIDS, http://www.healio. com/infectious-disease/ antimicrobials/news/in-the-journals/ experts-call-for-response-to-antibiotic-resistance-similar-tounaids. (accessed 12.12.20)

Heiko,Von, B. and Reinhard, M. 2005. Antimicrobial resistance of Escherichia coli and therapeutic implications. Int. J. Med Microbiol., 295(6-7): 503.

Kakkar, M., Walia, K., Vong, S., Chatterjee, P. and Sharma, A. 2017. Antibiotic resistance and its containment in India. B.M.J., 358: 2687.

Livermoore, D.M. and Paterson, D.L. 2006. Pocket guide to extended spectrum $\beta$-lactamases in resistance. New Delhi: Springer (India) Private Limited.

Magiorakos, A.P., Srinivasan, A., Carey, R.B., et al. 2012. Multidrug resistant, extensively drug-resistant and pandrugresistant bacteria: an international expert proposal for interim standard definitions for acquired resistance. Clin Microbiol Infect., 18(3): 268-281.

McKenna, M. 2013. Antibiotic resistance: the last resort. Nature, 25;499(7459): 394-6.

Morse, S.A., Johnson, S.R., Biddle, J.W. et al. 1986. High-level tetracycline resistance in Neisseria gonorrhoeae is result of acquisition of streptococcal tetM determinant. Antimicrob. Agents Chemother., 30: 664-670.

O'Donoghue, C. and Kyne, L. 2011. Update on Clostridium difficile infection. Curr. Opin. Gastrenterol., 27: 38.

Paterson, D.L. and Bonomo, R.A. 2005. Extended-spectrum $\beta$-lactamases: A clinical update. Clin. Microbiol. Rev., 18: 657-86.

Peleg, A.Y., Seifert, H. and Paterson, D.L. 2008. Acinetobacter baumannii: emergence of a successful pathogen. Clin. Microbiol Rev., 21: 538-582.

Peng, Z., Dazhi, Jin, Kim., H.B., Charles, W. Stratton, Bin Wu, Yi-Wei Tang and Xingmin Su. 017. Update on Antimicrobial Resistance in Clostridium difficile: Resistance Mechanisms and Antimicrobial Susceptibility Testing. J. Clin. Microbiol., 55(7): 1998-2008.

Phillips, I. 1976. Beta-lactamase-producing, penicillin-resistant gonococcus. Lancet, 2: 656-657.

Reinthaler, F.F., Posch, J., Feierl, G., Wüst, G., Haas, D., Ruckenbauer, G., Mascher, F. and Marth, E. 2003. Antibiotic resistance of E. coli in sewage and sludge. Water Res., 37: $1685-1690$.

Rupinder, B. Geeta, W. and Shikha, J. 2013. Prevalence of extended Spectrum $\beta$-lactamases in multidrug resistant strains of gram Negative Bacilli. J. Acad. Indus. Res., 1(9): 558

Schmidt, E.F., Smith, D.A. and Ryder, S.W.2007. Communicating the risk and benefit of medicines. Drug Discov. Today, 12: 355-364.

Spigaglia, P., Mastrantonio, P. and Barbanti, F. 2018. Antibiotic Resistances of Clostridium difficile. Adv. Exp. Med. Biol., 1050: $137-159$.

Suay-Garcia, B. and Pérez-Gracia, M.T. 2017. Drug-Resistant Neisseria gonorrhoeae: Latest developments. Eur. J. Clin. Microbiol. Infect. Dis., 36: 1065-1071.

Tacconelli, E., Carrara, E., Savoldi, A., Harbarth, S., Mendelson, M., Monnet, D.L., Pulci ni, C., Kahlmeter, G., Kluytmans, J., Carmeli, Y., Ouellette, M., Outterson, K., Patel, J., Cavaleri, M., Cox, E.M., Houchens, C.R., Grayson, M.L., Hansen, P., Singh, N., Theuretzbachr, U. and Magrini, N. 2018. WHO Pathogens Priority List Working Group. Discovery, research, and development of new antibiotics: the WHO priority list of antibiotic-resistant bacteria and tuberculosis. Lancet Infect Dis., 18: 318-327.

Tchesnokova, V., Riddell, K., Scholes, D., Johnson, J.R. and Sokurenko, E.V. 2019. Th Uropathogenic Escherichia coli Subclone Sequence Type 131-H30 Is Responsible for Most Antibiotic Prescription Errors at an Urgent Care Clinic. Clin. Infect Dis., 68(5): 781-787.

Ventola, C.L. 2015. The antibiotic resistance crisis: part 1: causes and threats. PT., 40(4): 277-283.

Walsh, C. and Wright, G. 2005. Introduction: antibiotic resistance. Chem. Rev., 105: 391- 394.

WHO. 2011. Tackling antibiotic resistance from a food safety perspective in Europe, available at: euro.who.int/_data/ assets/pdf_file/0005/136454/ e94889.pdf.

Wong, D., Nielsen, T.B., Bonomo, R.A., Pantapalangkoor, P., Luna, B. and Spellberg, B. 2017. Clinical and pathophysiological overview of Acinetobacter infections: a century of challenges. Clin Microbiol Rev., 30: 409- 447.

World Health Organisation. 2015. Global Action Plan on Antimicrobial Resistance. World Health Organization; Geneva, Switzerland: 2015. 
World Health Organization Draft Global Action Plan on Antimicrobial Resistance. 2014. Draft for Consultation with Member States, October 2014. [(Accessed on 30 November 2018)]; Availableonline: http://www.who.int/drugresistance/ AMR_DR AFT_GAP_1_O ct_2014_for_MS_consultation. pdf.

World Health Organization. 2017. Central Asian and Eastern European surveillance of antimicrobial resistance. Annual report 2017. World Health Organization Regional Office for Europe, Copenhagen, Denmark.
Zwald, A.G., Ruegg, P.L., Kaneene, J.B., Warnick, L.D., Wells, S.J., Fossler, C. and Halbert, L.W. 2004. Management Practices and Reported Antimicrobial Usage on Conventional and Organic Dairy Farms. J. Dairy Sci., 87(1): 191-201 
